\title{
Obstructive jaundice and cholangitis due to Ascariasis
}

Pragatheswaran P, MBBS ${ }^{1}$, de Silva W.D.D, MBBS, MD, MRCS'2, Abeysekera W.Y.M MBBS $^{1}$, Banagala A.S.K, MBBS, MS, FRCSEd ${ }^{3}$

1 Surgical Registrar, Colombo South Teaching Hospital; Kalubowila.

2 Senior Registrar, Colombo South Teahing Hospital, Kalubowila.

3 Consultant Surgeon, Colombo South Teaching Hospital, Kalubowila.

Key words: Ascariasis; Biliary obstruction;

Cholangitis.

\section{Introduction}

Biliary complications following ascarid round worm infestation is a relatively uncommon presentation of this common parasitic disease. We discuss the case of a patient presenting with acute cholangitis due to biliary ascariasis.

\section{Case report}

A 52 year old previously heảlthy male presented with a history of fever with chills, associated with severe colicky upper abdominal pain, vomiting and of the passage of dark coloured urine. On physical examination he was found to be acutely ill, febrile, icteric and dehydrated. Severe tenderness was noted over epigastric region.

Investigations revealed neutrophil leucocytosis (21190/mm3, 90\% neutrophils), elevated liver transaminases (AST 304U/L, ALT 337U/L) \& serum bilirubin (total- $28 \mathrm{mg} / \mathrm{dl}$, direct bilirubin $15 \mathrm{mg} / \mathrm{dl}$ ).

An ultrasound scan suggested acute cholecystitis with cholelithiasis. There was no evidence of intra or extrahepatic duct dilatation.

An ERCP was performed and on duodenal intubation, revealed a large ascaris worm hanging out through the major papilla (figure 1) with the head and two thirds of the worm inside the CBD. The $15 \mathrm{~cm}$ long worm was removed via the endoscope (figure 2). The patient made a remarkable recovery following removal of the worm. He was treated with oral antihelminthics and underwent cholecystectomy two weeks later for the co-existing gallstone disease. He continues to remain well.

Correspondence: P.Pragatheswaran, Surgical Registrar, Colombo South Teaching Hospital, Kalubowila.

Email: ppragathes@yahoo.com

The Sri Lanka Journal of Surgery 2011; 29(2):106-107.

\section{Discussion}

Infestation of the gastrointestinal tract by the adult roundworm Ascaris lumbricoides is one of the most common of human parasitic diseases with infestation of up to $25 \%$ of the world population [1]. Ascariasis has a long history of endemicity in Sri Lanka, although successful public health initiatives have decreased infestation rates significantly. Recent reports indicate the prevalence of round worm infestation is around 4\% among primary school children in the Western Province [2]. Hepato-biliary ascariasis caused by the migration of the worm in to the biliary tract is a relatively uncommon complication of this disease. However in areas of high endemicity such as the Kashmir valley and Syria it is a significant cause of biliary system morbidity [3-4]. Risk factors for migration include previous cholecystectomy, sphincterotomy and pregnancy [5]. Presentations of biliary ascariasis include biliary colic, cholecystitis, cholangitis, pancreatitis, gallstone formation, strictures and hepatic abscess [5]. In this case, our patient presented with symptoms of severe cholangitis.

Diagnosis is based on clinical suspicion and is usually achieved by ultrasonography. On ultrasound, the worms have a typically echogenic non-shadowing tubular structure with a relatively hypoechoic centre. The presence of a 'bulls-eye echo' on transverse sections is another classical sign [5]. In this instance however, ultrasound failed to demonstrate the worm.

According to large case series, conservative 


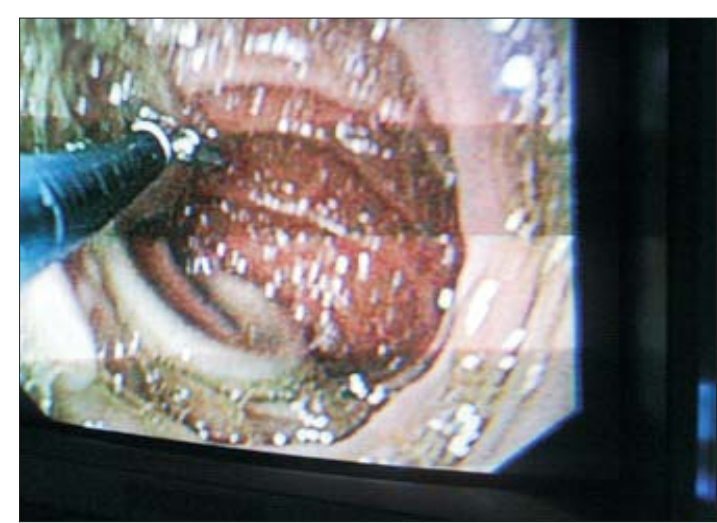

Figure 1: ERCP view: A large ascaris worm hanging out through the major papilla.

management with oral antihelminthics and analgesics is successful in up to $90 \%$ of cases [3, 4]. However in the presence of life-threatening complications such as cholangitis or pancreatitis, intervention becomes necessary. Endoscopy is the mainstay of interventional management and endoscopic worm removal via forceps leads to rapid resolution of symptoms. Worms completely within the biliary tree require extraction with biliary balloons or dormia basket. In this case, successful endoscopic removal facilitated dramatic recovery.

\section{References}

1. Shah OJ, Zargar SA, Robbani I. Biliary ascariasis. WorldJournal of Surgery 2006; 30:1500-1506.

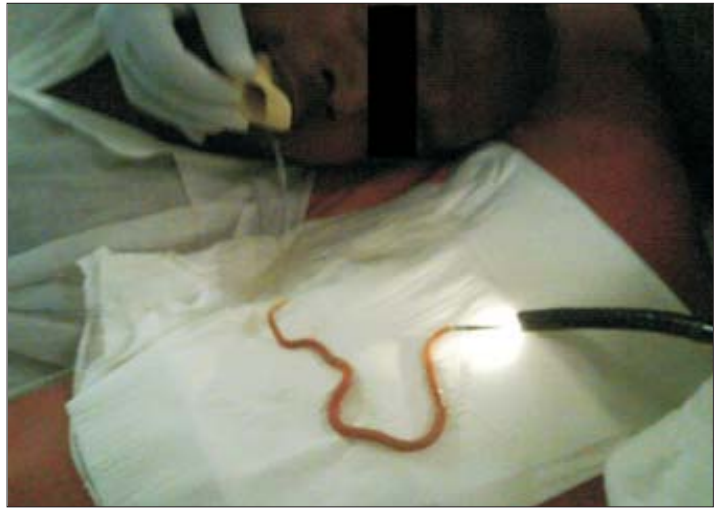

Figure 2: Removed worm.

2. Gunawardena NK, Amarasekera NDDM, Pathmeswaran A, de Silva NR. Effect of repeated mass chemotherapy for filariasis control on soiltransmitted helminth infections in Sri Lanka. Ceylon Medical Journal 2008; 53:13-6.

3. Khuroo MS, Zargar SA, Mahajan R. Hepatobiliary and pancreatic ascariasis in India. Lancet 1990; 335:1503-6.

4. Sandouk F, Haffar S, Zada MM, Graham DY, Anand BS. Pancreatic-biliary ascariasis: Experience of 300 cases. American Journal of Gastroenterology 1997; 92:2264-7.

5. Rana SS, Bhasin DK, Nanda M, Singh K. Parasitic infestations of the biliary tract. Current Gastroenterology Reports 2007; 9:156-164.

\section{Key Learning Points}

- Ascaris worm migration to the biliary tract is a rare cause of obstructive jaundice and cholangitis.

- An ultrasound scan may be diagnostic if a high index of suspicion is maintained.

- Endoscopic worm extraction is the procedure of choice. 